\title{
The role of exosomal PD-L1 in tumor progression and immunotherapy
}

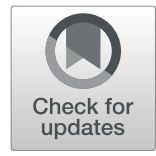

Feiting $\mathrm{Xie}^{1,2}$, Mengxue $\mathrm{Xu}^{1,2}$, Jian $\mathrm{Lu}^{1,2}$, Lingxiang Mao ${ }^{1^{*}}$ and Shengjun Wang ${ }^{1,2^{*}}$ (D)

\begin{abstract}
Programmed death ligand 1 (PD-L1), a type I transmembrane protein, binds to its receptor PD-1 to suppress the activation of T cells, thereby maintaining immunological homeostasis. In contrast, tumor cells highly express PD-L1, which binds to receptor PD-1 expressed on activated T cells, leading to immune escape. Anti-PD-1/PD-L1 immune checkpoint therapy blocks the binding of PD-1/PD-L1 to reinvigorate the exhausted T cells, thereby inhibiting tumor growth. Exosomes are biologically active lipid-bilayer nanovesicles secreted by various cell types that mediate intercellular signal communication. Numerous studies have shown that tumor cells are able to promote tumor epithelial-mesenchymal transition, angiogenesis, and immune escape by releasing exosomes. Recent studies imply that tumor-derived exosomes could carry PD-L1 in the same membrane topology as the cell surface, thereby resisting immune checkpoint therapy. In this review, we mainly discuss the role of exosomes in the regulation of tumor progression and the potential resistance mechanism to immunotherapy via exosomal PD-L1. In addition, we propose that exosomal PD-L1 may have the potential to be a target to overcome resistance to anti-PD-1/PD-L1 antibody therapy.
\end{abstract}

Keywords: Exosomes, PD-L1, PD-1, Tumor immunity, Biomarker

\section{Background}

Programmed death ligand 1 (PD-L1), also known as cluster of differentiation 274 (CD274) or B7 homolog (B7 homolog 1, B7-H1), is a type I transmembrane protein of 290 amino acids encoded by the CD274 gene and consisting of immunoglobulin V-like and C-like extracellular domains [1]. PD-L1 is widely expressed on various cell types, mainly in tumor cells, monocytes, macrophages, natural killer (NK) cells, dendritic cells (DCs), and activated $\mathrm{T}$ cells and also on immune-privileged sites such as the brain, cornea, and retina [2]. In normal physiological conditions, the activation of the PD-1/PDL1 signaling pathway is closely related to the induction and maintenance of peripheral tolerance, maintenance of $\mathrm{T}$ cell immune homeostasis, avoiding hyperactivation and protecting against immune-mediated tissue damage [3]. In disease states, PD-L1 interacts with its receptor programmed death 1 (PD-1), transmitting a negative signal to control a series of processes of $\mathrm{T}$ cell-mediated

\footnotetext{
* Correspondence: maolingxiang@aliyun.com; sjwjs@ujs.edu.cn 'Department of Laboratory Medicine, The Affiliated People's Hospital, Jiangsu University, Zhenjiang 212013, China

Full list of author information is available at the end of the article
}

cellular immune responses, including priming, growth, proliferation and apoptosis, and functional maturation [4]. Recently, research studies have found that the activation of the PD-1/PD-L1 signaling pathway can arrest the $\mathrm{T}$ cell cycle at the G1 phase rather than directly causing apoptosis [5]. In addition to mediating $\mathrm{T}$ cell-intrinsic inhibitory effects, the PD-1/PD-L1 signaling pathway could also induce the inhibition of $\mathrm{T}$ cell responses by inducible Tregs (iTregs) [6].

Recent studies on extracellular vesicles have demonstrated that exosomes are biologically active lipid-bilayer nanovesicles $(30-120 \mathrm{~nm})$ secreted by almost all types of normal or diseased cells through endosomal pathways into the extracellular space to transport "cargo" to the target cells to mediate intercellular signal communication [7-10]. The "cargo" is mainly composed of cytosolic proteins, microRNAs, circular RNAs, long noncoding RNAs, lipids, DNA, cytokines, transcription factor receptors and other molecules [11]. Research has shown that normal or diseased cells can secrete exosomal miRNAs to affect neighboring cells and/or distant target cells [12]. Noncoding RNAs in exosomes could influence the expression of tumor-related genes to cooperate in the

(C) The Author(s). 2019 Open Access This article is distributed under the terms of the Creative Commons Attribution 4.0 International License (http://creativecommons.org/licenses/by/4.0/), which permits unrestricted use, distribution, and 
cancer-causing process [13]. A rapidly growing number of studies have shown that exosomes participate in multiple physiological and pathological processes, especially the development of cancers.

Exosomes are widely distributed in body fluids such as saliva, plasma, urine, breast milk, and amniotic fluid both in cancer and noncancer cells from tumor patients [14]. Emerging evidence has shown that the exosomes secreted by tumor cells carry bioactive PD-L1 on their surface and can suppress the immune response [15]. Metastatic melanoma-derived exosomes, which are stimulated by interferon- $\gamma$ (IFN- $\gamma$ ), expressed more PD-L1 on these vesicles and inhibited antitumor responses [16]. In this review, we mainly discuss the role of exosomes in the regulation of tumor immunity and the potential resistance mechanism to immunotherapy via exosomes that express PD-L1 on their surface (the same membrane topology as cell surface PD-L1).

\section{The regulatory role of exosomes in tumor progression}

\section{Exosomes inhibit tumor progression}

In the tumor microenvironment, exosomes may mediate intracellular communication and immune regulation. Tumor-derived exosomes (TEXs) and immune cell-derived exosomes (IEXs) have been demonstrated to activate immune responses by transferring antigens to antigen presenting cells (APCs), which results in the activation of $\mathrm{CD} 4^{+} \mathrm{T}$ cells and $\mathrm{CD} 8^{+} \mathrm{T}$ cells, thereby enhancing the anti-tumor responses and leading to the inhibition of tumor progression [17]. Additionally, an alternative mode of action has also been described that involves inducing the apoptosis of tumor cells [18]. The latest research has found that TEXs and IEXs could be applied to oncotherapy as monitoring markers instead of detecting $\mathrm{T}$ lymphocytes and taking tumor biopsies [19]. In the following description, we introduce the anti-tumor effect of TEXs and IEXs. (Tables 1 and 2).

Considering the origin of exosomes, TEXs may contain some tumor-associated antigens, including melan A, carcinoembryonic antigen and mesothelin $[39,40]$. Thus, TEXs
Table 2 The regulatory role of immune cell-derived exosomes in tumor progression

\begin{tabular}{|c|c|c|c|c|c|c|c|}
\hline \multirow{2}{*}{$\begin{array}{l}\text { Origin of } \\
\text { Exosomes }\end{array}$} & \multicolumn{5}{|c|}{ Target cells } & \multirow{2}{*}{$\begin{array}{l}\text { Tumor } \\
\text { progression }\end{array}$} & \multirow[t]{2}{*}{ References } \\
\hline & $\overline{\mathrm{DC}}$ & $\mathrm{CD}^{+} \mathrm{T}$ & $\mathrm{CTL}$ & Treg & $\overline{T h 17}$ & & \\
\hline$\overline{D C}$ & & $\uparrow$ & $\uparrow$ & $\downarrow$ & & $\downarrow$ & [32] \\
\hline $\mathrm{CD}^{+} \mathrm{T}$ cell & & & $\downarrow$ & & & $\uparrow$ & [33] \\
\hline $\mathrm{CD}^{+} \mathrm{T}$ cell & $\downarrow$ & & $\uparrow$ & & & $\uparrow \downarrow$ & {$[7,34]$} \\
\hline Macrophage & & $\uparrow$ & & & & $\downarrow$ & [35] \\
\hline TAM & $\downarrow$ & & & $\uparrow$ & $\uparrow$ & $\uparrow$ & [32] \\
\hline Mast cell & $\uparrow$ & $\uparrow$ & $\uparrow$ & & & $\downarrow$ & [35] \\
\hline B cell & $\uparrow$ & $\uparrow$ & $\downarrow \uparrow$ & & & $\downarrow \uparrow$ & {$[35,36]$} \\
\hline MDSC & & & $\downarrow$ & & & $\uparrow$ & [37] \\
\hline Treg & & & $\downarrow$ & & & $\uparrow$ & [38] \\
\hline
\end{tabular}

Tumor progression includes: $\uparrow$ promotion, $\downarrow$ inhibition

DC Dendritic cell, MSC Mesenchymal stem cell, CTL Cytotoxic T lymphocyte, NK Natural killer, M2 M2 macrophage, TAM Tumor-associated macrophages cell,

Treg Regulatory T cell, MDSC Myeloid-derived suppressor cell, Th T helper

could be used to form a pool of tumor antigens to stimulate the anti-tumor response. Currently, TEXs have been widely used for the induction of anti-tumor responses in both murine models and clinical trials. A recent study reported that exosomes derived from heat-stressed tumor cells could induce the production of IL- 6 by DCs and marcophage, which switches regulatory $\mathrm{T}$ cell into Th17 in tumor microenviroment in a HSP-70 dependent manner [20]. DCs have been proven to be a target for TEXs to enhance anti-tumor responses [21]. Research has found that EG7 tumor cell-derived exosomes transfer parental cell-associated antigen OVA and pMHC-I to DCs, which stimulate a stronger proliferation and differentiation of cytotoxic T lymphocytes (CTL) and generating a more robust OVA-specific antitumor immunity than control ones. Similar results were obtained in hepatocellular carcinoma (HCC) models and in other studies [21, 22]. Simultaneously, exosomes from TGF- $\beta$-silenced leukemia cells decrease the secretion of TGF- $\beta$ by DCs and effectively promote their maturation and function. Additionally, DCs carrying these exosomes facilitated the proliferation of $\mathrm{CD}^{+} \mathrm{T}$ cells and enhanced the antigen-specific CTL

Table 1 The regulatory role of tumor cell-derived exosomes in tumor progression

\begin{tabular}{|c|c|c|c|c|c|c|c|c|c|}
\hline \multirow[t]{2}{*}{ Origin of Exosomes } & \multicolumn{7}{|c|}{ Target cells } & \multirow{2}{*}{$\begin{array}{l}\text { Tumor } \\
\text { progression }\end{array}$} & \multirow[t]{2}{*}{ References } \\
\hline & $\overline{D C}$ & CTL & Treg & Th17 & M2 & NK & Monocyte & & \\
\hline Heat-stressed tumor cell & $\uparrow$ & & $\downarrow$ & $\uparrow$ & & & & $\downarrow$ & {$[20]$} \\
\hline Hepatocellular carcinoma cell & $\uparrow$ & $\uparrow \downarrow$ & & & $\uparrow$ & $\downarrow$ & & $\uparrow \downarrow$ & {$[21-24]$} \\
\hline Malignant glioma cell & $\uparrow$ & $\uparrow$ & & & & & & $\downarrow$ & {$[25]$} \\
\hline Leukemia cell & $\uparrow$ & $\uparrow$ & & & & $\uparrow$ & & $\downarrow$ & {$[26,27]$} \\
\hline RenCa cell & & $\uparrow$ & & & & & & $\downarrow$ & {$[28]$} \\
\hline Gastric cancer cell & & $\downarrow$ & & & & & $\uparrow$ & $\uparrow$ & {$[29]$} \\
\hline Melanoma cell & & $\downarrow$ & & & & & & $\uparrow$ & {$[16,30,31]$} \\
\hline
\end{tabular}

Tumor progression includes: $\uparrow$ promotion, $\downarrow$ inhibition 
responses [26, 27]. Interestingly, TEXs which exert a stable antitumor response are mostly based on targeting DCs. These provide a new idea for our future research.

It has been reported that IEXs also contribute to enhancing the anti-tumor response. In addition, IEXs could alter the microenvironment suitable for tumors to suppress tumor growth. Recently, DC-derived exosomes (DEXs) have been recognized as a new class of vaccines for tumor therapy $[35,41]$. In this research, $\mathrm{Lu}$ and coworkers found that exosomes derived from a-fetoprotein (AFP)-expressing DCs could promote the antigenspecific immune response through elevating the levels of IFN- $\gamma$ and interleukin- 2 and reducing the expression of interleukin-10 and TGF- $\beta$. Activated $\mathrm{CD}^{+} \mathrm{T}$ cellderived extracellular vesicles are able to directly target mesenchymal tumor stromal cells to prevent tumor invasion and metastasis [34]. Exosomes released by NK cells have also been identified as having therapeutic effects. Both in vitro and in vivo experiments revealed that NK cell-derived exosomes could suppress the development of melanoma via their contents of TNF- $\alpha$, perforin and FasL [42]. In neuroblastoma (NB) tumors, exosomes derived from NK cells pretreated with NB cells increased the expression of natural killer cell receptors and enhanced the cytotoxicity of NK cells against NB tumors [43].

In addition to the exosomes mentioned above, exosomes derived from mesenchymal stem cells (MSCs) have also been reported to restrain tumor development [44]. MSC-derived exosomes have potent regulatory effects on immune responses involving different immune cells, such as $\mathrm{T}$ cells and B cells [45]. Researchers have demonstrated that human adipose MSC-derived exosomes inhibit the proliferation and colony formation ability of A2780 and SKOV-3 human ovarian cancer cells via inducing the expression of BAX and CASP3/9 while reducing the levels of BCL2 [46]. Interestingly, researchers have obtained similar results from human umbilical cord MSC-derived extracellular vesicles (EVs) [47].

\section{Exosomes promote tumor progression}

Despite exosomes having anti-tumor effects as mentioned above, more studies have focused on their effects in promoting tumor progression [48]. The growth of tumor is associated with various growth factor receptors and signaling pathways. These receptors triggers downstream signaling pathways via Akt, PKC/PKB and ERK kinase pathways through the activation or phosphorylation of intracellular kinase domain which leads to tumor cell proliferation and migration.

In NB tumors, TEXs promote tumor proliferation and migration by decreasing the expression of NEDD4 by hsamiR199a-3p [49]. TEXs derived from the Lewis lung cancer model and $4 \mathrm{~T} 1$ breast cancer model inhibit the differentiation of myeloid precursors into DCs and their maturation by increasing the expression of immunosuppressive markers PD-L1 to trigger inhibitor signals [50]. Moreover, studies have reported that gastric cancer-derived exosomes can induce monocytes to differentiate into PD- $1^{+}$ tumor-associated macrophages (TAMs), which can effectively suppress anti-tumor responses by triggering the PD-1/ PD-Ls signaling pathway [51]. Exosomes derived from oral squamous cell carcinoma could upregulate the expression of PD-L1 on myeloid-derived suppressor cells (MDSCs) to induce $\mathrm{y} \delta \mathrm{T}$ cell exhaustion through an exosome miR-21/ PTEN/PD-L1 pathway [52]. Similar results have been obtained in hepatocellular carcinoma (HCC) cells. Liu J and colleagues discovered that exosomes released from HCC cells upregulated the expression of PD-L1 in macrophages to inhibit T-cell function through a miR-23a/PTEN/AKT regulation axis [53]. In addition, exosomes obtained from melatonin-treated hepatocellular carcinoma cells were able to downregulate the expression of PD-L1 and the secretion of cytokines (IL-6, IL-1 $\beta$, IL-10, and TNF- $\alpha$ ) in macrophages [54]. In light of this, we surmise that miRNAs contained in tumor-derived exosomes may facilitate the growth and metastasis of tumors [55].

IEXs and exosomes secreted by other cells have also been reported to facilitate the growth and metastasis of tumors. Exosomes derived from activated $\mathrm{CD}^{+} \mathrm{T}$ cells can promote the invasion of tumor cells via the Fas/FasL pathway [7]. However, another study has shown that activated $\mathrm{T}$ cell-derived exosomes promote tumor invasion and metastasis through the ERK and NF- $\mathrm{BB}$ pathways. Exosomes released by activated OVA-specific $\mathrm{CD}_{4}^{+} \mathrm{T}$ cells can suppress the cytotoxicity of DC-stimulated $\mathrm{CD}^{+} \mathrm{T}$ cells via pMHC II/TCR and CD54/LFA-1 interactions. Recently, a study described the functions of EVs derived from $B$ cells in anti-tumor responses [36]. $\mathrm{CD}_{39}{ }^{+} \mathrm{CD} 3^{+} \mathrm{EVs}$ released by tumor $\mathrm{B}$ cells hydrolyze ATP and AMP from tumor cells into adenosine to inhibit the proliferation of $\mathrm{CD}^{+} \mathrm{T}$ cells. In a clinical trial, higher CD19 ${ }^{+}$EVs in tumor patients' serum were associated with poor efficacy of chemotherapy. These results indicate that exosomes may not have the characteristics of the parent cells [33]. In osteosarcoma and gastric cancer patients, researchers have found that human bone marrow MSC-derived exosomes (MSC-exo) could promote tumor progression through the activation of the Hedgehog signaling pathway [56]. Our data shown that exosomal S100A9 from granulocytic myeloid-derived suppressor cells promotes CRC cell stemness and growth [9].

Whether pro-tumor or anti-tumor function of TEXs and IEXs, these researches state the importance of them in tumor progression and define emergence of new paradigms in tumor cell biology. In terms of tumor inhibition, IEXs exert anti-tumor response primarily through 
their contents, such as TNF- $\alpha$, perforin, FasL and so forth. However, TEXs activate the anti-tumor immune response mainly by presenting tumor antigens to DCs. In terms of tumor promotion, the molecules expressed on IEXs, such as FasL and pMHC II, interact with T cells to trigger co-inhibitor signals and promote apoptosis. TEXs have the similar effect as IEXs. The contents contained in TEXs have the ability to up-regulate the expression of PD-L1 on myeloid cells to inhibit the function of $\mathrm{T}$ cells through PD-1/PD-L1 signaling pathway. And exosomal MHC I could promote this inhibit function by inducing the first signal activation of $\mathrm{T}$ cells. Recent studies have demonstrated that exosomes can carry PD-L1 on their surface and have been proven to play a key role in tumor development [57-59]. Because of their remarkable immunosuppressive function, exosomes may be a cancer therapy target in the future.

\section{Upregulated expression of PD-L1 induces immune escape to promote tumor development}

The function of PD-L1 expressed on cells in suppressing anti-tumor immune response

The expression of PD-L1 is upregulated in different types of cancer and noncancer cells, such as macrophages, monocytes, and tumor cells. Its receptor PD-1 is mainly expressed on activated $\mathrm{CD}^{+}$and $\mathrm{CD}^{+} \mathrm{T}$ cells, B cells, monocytes and MDSCs [60]. One of the important mechanisms of tumor immune evasion is that PD-L1 expressed on the tumor cell surface binds to PD-1 on T cells, leading to an immune checkpoint response. Under normal conditions, activated antigen-specific cytotoxic $\mathrm{T}$ lymphocytes can recognize and directly respond to tumor cells. However, PD-L1-overexpressing tumor cells have the exceptional capability to survive, escape the immune system surveillance and then invade neighboring tissue [61].

In recent clinical studies, researchers have found that the high expression of PD-L1 in patients with triplenegative breast cancer tissue may be associated with poor prognosis [62]. Recent studies have found that tumor cells have the potential to control the molecular mechanism (depletion of HIP1R) of lysosomal degradation of PD-L1, leading to its intracellular accumulation and inducing immune tolerance. The peptide PD-LYSO successfully targets PD-L1 expressed by tumor cells to lysosomes for degradation, taking the place of the depleted HIP1R [63]. Moreover, Chen J. et al. demonstrated that IFN- $\gamma$, which is secreted by inflammatory cells such as macrophages and NK cells, can upregulate PD-L1 expression on tumor cells $[64,65]$. With upregulated expression of PD-L1, tumor cells mediate adaptive resistance to the IFN- $\gamma$ released by cytotoxic $\mathrm{T}$ lymphocytes, creating a vicious circle that exacerbates the disease [65]. In gastric cancers, several previous reports have demonstrated that PD-L1 expression can range from 25 to $65 \%$ on tumor cells [51].

Simultaneously, studies have shown that the infiltration of immune cells in the tumor microenvironment may be associated with the presentation of PD-L1 on their surface [66]. In the tumor hypoxic microenvironment, the expression of PD-L1 on MDSCs is increased, and the changing level of PD-L1 can be used to evaluate the efficacy of hyperoxic therapy [67]. Moreover, TAMs, which overexpress PD-L1 on the cell surface, may suppress the function of cytotoxic $\mathrm{T}$ lymphocytes. Another hypothesis has suggested that the TAM receptors Tyro3, Axl, and Mertk could upregulate the expression of PDL1 on a breast cancer cell line [68]. Furthermore, IFN- $\gamma$ could facilitate the level of PD-L1 expressed on exosomes released by melanoma cells that we will discuss later in this review. Thus, the potential effects of PD-L1 overexpression on different cell types are not clearly understood.

\section{Exosomal PD-L1 is involved in inducing immune escape to promote tumor progression}

A previous study verified that PD-L1 exists in exosomes from human urine or plasma [69]. Studies have confirmed that the levels of PD-L1 expressed on exosomes, but not soluble PD-L1, correlate with disease progression in head and neck squamous cell carcinoma (HNSC C) [58]. Flow cytometry and immunofluorescence results suggested that PD-L1 is present not only on the surface of vesicles but also within vesicle-like structures [70]. PD-L1 could be specifically packaged into exosomes with the help of both Rab27a and nSMase2. Knocking out Rab27a and nSMase 2 in tumor cells causes a loss of PD-L1 in the secreted fraction. Researchers found that exosomal PD-L1 has the same membrane topology as cell surface PD-L1 using an enzyme-linked immunosorbent assay [16]. Recent reports have visually pointed out that exosomal PD-L1 can transfer to other cells with or without PD-L1 expression in a dose-dependent manner in vitro and in vivo [71]. As the number of studies of PD-L1 on exosomes increases, its critical role in research has been gradually revealed. It was worth mentioning that the results referred below are all about tumorderived exosomal PD-L1, although the expression of PD-L1 on myeloid cell is critical in inducing immune suppression, there is no published report studying about the myeloid cell- derived exosomal PD-L1.

Glioblastoma, a local and systemic immunosuppressive neoplasm, is able to secrete inflammatory cytokines, initiating the immune checkpoint response. In addition, EVs released by glioblastoma stem-like cells (GSCs) express PD-L1 on their surface and play a critical role in mediating the inhibition of both $\mathrm{CD}^{+}$and $\mathrm{CD}^{+} \mathrm{T}$ cell activation and proliferation by an anti-CD3 mAb [72]. In 
the 4-nitroquinoline 1-oxide-induced malignant oral/ esophageal injury model, exosomes carrying PD-L1 isolated from supernatants of murine or human HNSCC cell lines can hamper the infiltration of $\mathrm{CD}_{4}^{+} \mathrm{T}$ and $\mathrm{CD}{ }^{+} \mathrm{T}$ cells into the tumor microenvironment, thereby accelerating tumor progression [73]. Exosomal PD-L1 from TRAMP-C2 cells has no significant differences between Rab27a and PD-L1 knockout phenotypes in promoting tumor progression [74]. However, in MC38 tumor models, PD-L1 knockout plays a more critical role than Rab27a loss does. Thus, in different tumor models, the role of exosomal PD-L1 in promoting tumor progression is diverse.

To address whether the loss of exosomal PD-L1 would reverse the PD-L1-mediated immunosuppression in vivo and in vitro, researchers established a xenograft model with PD-L1 knockdown cell lines and then found that tumor growth was promoted by the exosomes released by parental cells. The same conclusion has been reached in 4 T1-PD-L1 (knockdown) tumor models. In the draining lymph nodes of mice injected with Rab27a-null or PD-L1-null TRAMP-C2 cells, the percentage of CD8positive cells is upregulated, and the proportion of cells expressing the exhaustion marker Tim3 is downregulated, while the proportion of cells expressing the activation markers granzyme B and Ki67 is increased. These results indicate that the loss of exosomal PD-L1 can relieve the inhibition of $\mathrm{T}$ cell activation in the draining lymph nodes. Moreover, exosomal PD-L1 plays an important role in not only immediate immune response but also memory ones. Mice injected with either Pd-11 or Rab27a null TRAMP-C2 cells on one flank not only suppresses growth of the local tumor cells but also blocks WT tumor cells rechallenged on the other flank in 90 days. This indicated that $\mathrm{T}$ cells derived from a xenograft model lacking exosomal PD-L1 possess the ability to exert a robust antitumor memory response resistant to the inhibitory effect of exosomal PD-L1. In addition, exosomal PD-L1 derived from other cancer cells such as colon and lung cancer cells has similar effects on $\mathrm{T}$ cell activation and tumor progression. (Table 3).
A recent study roughly divided solid tumors into 'hot' or 'cold' according to whether the tumors were surrounded by $\mathrm{T}$ cells or not [75-77]. In view of the function of exosomal PD-L1 mentioned above, we suspect that PD-L1 may have an effect on $\mathrm{T}$ cells migrating to the tumor site.

\section{Exosomal PD-L1 mediates resistance to immunotherapy by directly binding to anti-PD-L1 antibody}

In the past few decades, the immunotherapy of tumors has captured widespread attention in the medical community. Immunotherapy can be broadly divided into two different types: somatic immunotherapy and immune checkpoint inhibitor treatment. Immune checkpoint blockade treatments include anti-CTLA-4 mAb, antiPD-1 mAb, and anti-PD-L1 mAb [78]. It has been reported that the attractive system E1A-engineered mesenchymal stromal cell (MSC. E1A) can locally modify tumor cells to release CD3-HAC (PD-1), which can be bound to PD-L1-positive breast cancer cells. Therefore, the recruited $\mathrm{T}$ cells can exert their potential role in anti-tumor immunity [79]. A study has demonstrated that monoclonal antibodies against PD-1/PD-L1 demonstrate prominent effectiveness in patients with different types of cancers, such as non-small-cell lung cancer (NSCLC), HNSCC, melanoma, colorectal cancer, and breast cancer [80-84]. However, some solid tumors, such as prostate cancer, have been shown to be resistant to the treatment with anti-PD-L1 therapy [85]. The mechanism of therapeutic resistance among patients remains largely unknown. In view of the roles of exosomal PD-L1 in tumor progression, we asked whether exosomal PD-L1 could induce therapeutic resistance to antiPD-L1 antibody treatment.

It is widely known that PD-1/PD-L1 blockade could activate $\mathrm{T}$ cells, but little is known about the role of exosomal PD-L1 in the relatively low response rate of antiPD-L1/PD-1 therapy [86]. Pretreatment of glioblastoma EVs with anti-PD-1 receptor-blocking antibodies nearly reversed the suppression of $\mathrm{T}$ cells and prevented tumor progression [72]. Chen and colleagues have also found that PD-L1 on metastatic melanoma-derived exosomes

Table 3 The function of exosomal PD-L1 in tumor progression

\begin{tabular}{|c|c|c|c|}
\hline Type of tumor & Source of exosomes & Function & References \\
\hline Melanoma & Plasma & $\begin{array}{l}\text { Suppress the function of } \mathrm{CD}^{+} \mathrm{T} \text { cells and } \\
\text { cause failure of anti-PD-1 therapy }\end{array}$ & [16] \\
\hline Breast cancer & Tumor tissue & $\begin{array}{l}\text { Inhibit the secretion of granzyme B by cytotoxic } \\
\text { lymphocytes to promote tumor growth }\end{array}$ & [71] \\
\hline Prostate cancer & Tumor tissue & $\begin{array}{l}\text { Suppress the function of T cells in the draining } \\
\text { lymph node and block anti-PD-L1 antibodies }\end{array}$ & [70] \\
\hline $\begin{array}{l}\text { Head and neck squamous } \\
\text { cell carcinomas }\end{array}$ & Plasma & $\begin{array}{l}\text { Downregulate CD69 expression on effector T cells } \\
\text { to inhibit anti-tumor responses }\end{array}$ & [58] \\
\hline
\end{tabular}


inhibits the activation of $\mathrm{CD}^{+} \mathrm{T}$ cells and facilitates tumor growth, and these effects can be disrupted by anti-PD-1 antibody (aPD-1) therapy [16]. In a prostate cancer syngeneic model, mice were not responsive to anti-PD-L1 antibody (aPD-L1) treatment because of exosomal PD-L1. In 4 T1 tumor models, the accumulation of exosomal PD-L1 in the tumor microenvironment induced immunotherapy resistance by suppressing the secretion of granzyme B. Notably, knockdown of Rab27a in tumor cells significantly enhanced the efficiency of anti-PD-1 therapy and inhibited 4 T1 tumor growth [71]. In HNSCC patients, PD-L1 ${ }^{\text {high }}$ exosomes significantly inhibit $\mathrm{CD} 69$ on $\mathrm{CD}^{+} \mathrm{T}$ cells and can be blocked by anti-PD-1 antibodies [58]. These results indicate that exosomal PD-L1 plays an important role in anti-PD-L1/ PD-1 therapy. However, the concrete resistance mechanism of exosomal PD-L1 is largely unclear. Not only exosomal PD-L1 but also PD-L1 splicing variants play a crucial role in tumor progression. Cells from patients who secreted PD-L1 splicing variants were able to competitively bind to aPD-L1 antibody, which lead to therapeutic resistance [87]. Recent data implied that tumor- and regulatory $\mathrm{T}$ cell (Treg)-derived exosomes carrying CTLA-4 on their surface could interfere with immunotherapy with ipilimumab. These results suggest that the molecules carried on exosomes may be a novel mechanism for patients to generate therapeutic resistance and may be potential biomarkers for tumor diagnoses [19].

Although anti-PD-L1/PD-1 therapy has already been applied to clinical studies, questions remain to be further explored. Scientists have confirmed that after sequential use of PD-1 and PD-L1 antibodies, fatal myocarditis developed in a patient with brain metastatic lung adenocarcinoma [88]. Another research study demonstrated that anti-PD-1/PD-L1 treatment may offer a potential immunotherapy to cure Alzheimer's disease and dementia [89]. Although immune checkpoint blockade has fewer side effects than conventional chemotherapy and radiotherapy, its specific mechanism remains to be studied in detail [90]. (Fig. 1).

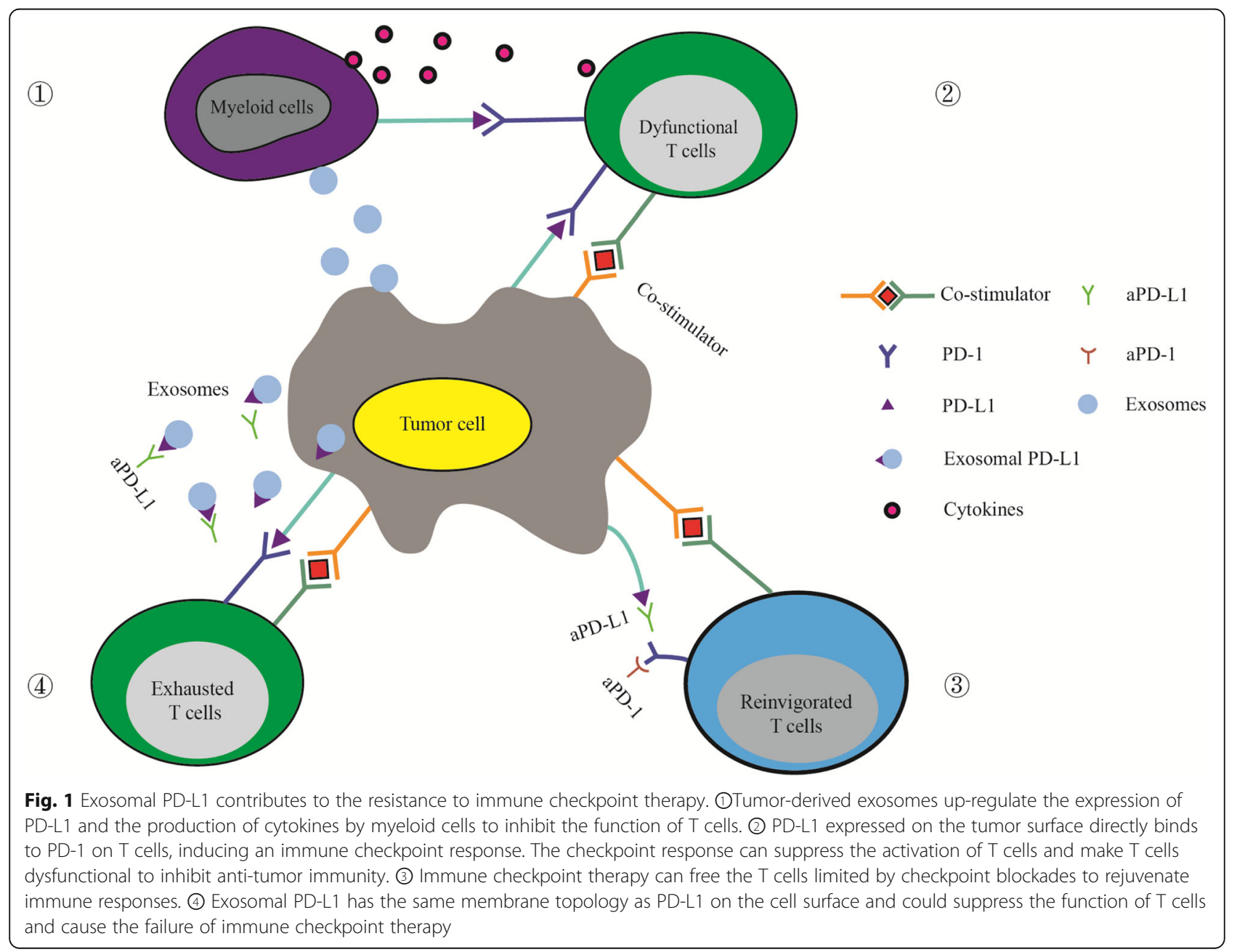




\section{Exosomal PD-L1 may act as a tumor biomarker}

Prior studies have focused on the use of exosomes as a genetic engineering vaccine targeting tumor cells, but an exponentially increasing number of studies has found that tumor-derived exosomes could be tumor diagnostic markers. In the past few decades, many researchers have committed to finding biomarkers in exosomes to specifically detect or predict diseases [91].

Exosomes can express various function-related molecules that are detected on the membranes of parent cells. The prognosis of head and neck cancer or melanoma patients varies with the different levels of circulating exosomal PD-L1. Before receiving treatment, the levels of circulating exosomal PD-L1 in patients who responded to anti-PD-1 antibodies were higher than those in nonresponders. The fold change of exosomal PD-L1 was more significant in responders than nonresponders after undergoing therapy. Hence, exosomal PD-L1 may be a biomarker for clinical immunotherapy to detect its levels in patient blood prior to therapy. Another study has demonstrated that the detection of PD-L1 $1^{+}$exosomes in serum was correlated with poor prognosis in patients with pancreatic ductal adenocarcinoma [92]. Further study has implied that miR-21 contained in PD- $\mathrm{L}^{+}$exosomes has the potential to be a biomarker for distinguishing the differences between NSCLC patients and healthy controls [93]. Going forward, in the process of anti-PD-1/PD-L1 immune checkpoint therapy, we should take not only the cell surface PD-L1 but also the exosomal ones into account.

In short, the results mentioned above provide a theoretical basis for exosomal PD-L1 as a biomarker, but need to be expanded to make the liquid biopsy of exosomes possible.

\section{Conclusion and future perspective}

All together, both tumor-derived exosomes and immune cell-derived exosomes play dual roles in tumor progression. Although exosomes could inhibit cancerous growth, the purpose of completely tumor eradication has not been come true yet. Therefore, the exact reason and mechanism of it still remain to be explored.

The PD-1/PD-L1 pathway plays a critical role in maintaining the balance between immunological tolerance and autoimmunity, but it can also be a way for tumor cells to evade attacks by the host immune system. Various studies have demonstrated that exosomal PD-L1 could dock to target $\mathrm{T}$ cells to induce it anergy and apoptosis. However, the action modes and mechanisms of exosomal PD-L1 remain to be urgently further explored. Tumor cells can deliver exosomes to different cell types to achieve the purposes of assimilation, including tumor cells, tumor mesenchymal cells, macrophages and DCs. And studies have also implied that tumor-derived exosomes is closely associated with the formation of a pre-metastatic niche. So we take a wild suspect if the increasing expression of PD-L1 in TAM, MDSC, monocytes during tumorigenesis due to the receipt of excessive tumor-derived exosomal PD-L1. It is worth noting that the current research on exosomal PD-L1 is mainly focused on tumor sources. Although noncancer cells in the tumor microenvironment also highly expressed PD-L1, such as DC, MDSC, macrophage, etc., there is no knowledge of whether other cell-derived exosomes can exert influence on tumor progression by producing exosomal PD-L1. It may be a question worthy of further discussion. Researchers also pointed out MHC molecules expressed on exosomes play an essential part in tumor promotion by exosomal PD-L1. exosomal MHC I molecular interact with TCR enhanced the suppress function of exosomal PD-L1 to T cells. These might be perfectly justifiable reasons to illustrate on why exosomal PD-L1 exert a more robust immunosuppressive effects than the soluble form. These results also re-energised the questions whether other exosomal molecular develop synergistic effects of exosomal PD-L1.Based on previous findings, we hypothesis that these molecular antagonists may have the potential to work synergistically with immunotherapy to exert anti-tumor response. Patients treated with PD-1/PD-L1 blockade have significant individual differences. Exosomal PD-L1 is resistant to immunotherapy may be due to the low abundance relative to surface PD-L1. Thus, it would be vital to tease apart exosomal PD-L1 with current immune-checkpoint therapies and exploit a more effective small molecules targeting exosomal PD-L1. Alternatively, up-regulate the expression of PD-L1 on exosomes to make recognized by the delivered antibody being possible. Applying the theoretical basis of exosomal PD-L1 to clinical treatment still has a lot of work to do.

In short, exosomal PD-L1 has a vital function in tumor metastasis, immune escape, and immunotherapy, but we know nothing about whether the function of exosomal PD-L1 is cancer type-dependent or not. Further clarification of the role of exosomal PD-L1 in tumor progression contributes to the early diagnosis and treatment of cancer. Moreover, studies on applying exosomal PD-L1 in noninvasive "liquid biopsy" as a biomarker for cancer are frenetic investigation.

\footnotetext{
Abbreviations

APCs: Antigen presenting cells; CTL: Cytotoxic T lymphocyte; DCs: Dendritic cells; HCC: Hepatocellular carcinoma; HNSCC: Head and neck squamous cell carcinoma; IEXs: Immune cell-derived exosomes; IFN- $\gamma$ : Interferon- $\gamma$; MDSC: Myeloid-derived suppressor cell; MSC: Mesenchymal stem cell; NK: Natural killer; PD-1: Programmed death 1; PD-L1: Programmed death ligand 1; TAM: Tumor-associated macrophages cell; TEXs: Tumor-derived exosomes; Th: T helper cell; Treg: Regulatory T cell
} 


\section{Authors' contributions}

FX drafted the manuscript. MX and $J L$ discussed and revised the manuscript. SW and LM designed the study and drafted the manuscript. All authors read and approved the final manuscript.

\section{Funding}

This work was supported by the Jiangsu Province's Key Medical Talents Program (Grant No. ZDRCB2016018, QNRC2016453), the Specialized Project for Clinical Medicine of Jiangsu Province (Grant No. BL2014065), and Project Funded by the Priority Academic Program Development of Jiangsu Higher Education Institutions. The funding body had no role in the design of the study and collection, analysis, and interpretation of data or preparation of the manuscript.

\section{Availability of data and materials}

The material supporting the conclusion of this review has been included within the article.

\section{Ethics approval and consent to participate}

This is not applicable for this review.

\section{Consent for publication}

Not applicable.

\section{Competing interests}

The authors declare that they have no competing interests.

\section{Author details}

'Department of Laboratory Medicine, The Affiliated People's Hospital, Jiangsu University, Zhenjiang 212013, China. ${ }^{2}$ Department of Immunology, Jiangsu Key Laboratory of Laboratory Medicine, School of Medicine, Jiangsu University, Zhenjiang, China.

Received: 18 July 2019 Accepted: 12 September 2019 Published online: 23 October 2019

\section{References}

1. Shi L, Chen S, Yang L, Li Y. The role of PD-1 and PD-L1 in T-cell immune suppression in patients with hematological malignancies. J Hematol Oncol. 2013;6:74. https://doi.org/10.1186/1756-8722-6-74.

2. Francisco LM, et al. PD-L1 regulates the development, maintenance, and function of induced regulatory T cells. J Exp Med. 2009;206:3015-29. https:// doi.org/10.1084/jem.20090847.

3. Bardhan K, Anagnostou T, Boussiotis VA. The PD1:PD-L1/2 pathway from discovery to clinical implementation. Front Immunol. 2016;7:550. https://doi. org/10.3389/fimmu.2016.00550.

4. Kamimura N, Wolf AM, Iwai Y. Development of Cancer immunotherapy targeting the PD-1 pathway. J Nippon Med Sch. 2019:86:10-4. https://doi. org/10.1272/jnms.JNMS.2019_86-2.

5. Patsoukis N, et al. Selective effects of PD-1 on Akt and Ras pathways regulate molecular components of the cell cycle and inhibit T cell proliferation. Sci Signal. 2012;5:ra46. https://doi.org/10.1126/scisignal. 2002796.

6. Zhang SA, et al. Effect of EBI3 on radiation-induced immunosuppression of cervical cancer HeLa cells by regulating Treg cells through PD-1/PD-L1 pathway. Tumour Biol. 2017;39:1010428317692237. https://doi.org/10.1177/ 1010428317692237.

7. Cai Z, et al. Activated T cell exosomes promote tumor invasion via Fas signaling pathway. J Immunol. 2012;188:5954-61. https://doi.org/10.4049/ jimmunol.1103466.

8. Kalluri R. The biology and function of exosomes in cancer. J Clin Invest. 2016;126:1208-15. https://doi.org/10.1172/JC181135.

9. Wang $Y$, et al. Granulocytic myeloid-derived suppressor cells promote the stemness of colorectal cancer cells through exosomal S100A9. Adv Sci. 2019;6:1901278. https://doi.org/10.1002/advs.201901278.

10. Zhang L, Yu D. Exosomes in cancer development, metastasis, and immunity. Biochim Biophys Acta Rev Cancer. 2019;1871:455-68. https://doi.org/10. 1016/j.bbcan.2019.04.004.

11. Tkach $M$, Thery $C$. Communication by extracellular vesicles: where we are and where we need to go. Cell. 2016;164:1226-32. https://doi.org/10.1016/j. cell.2016.01.043.
12. Yang $F$, et al. Exosomal miRNAs and miRNA dysregulation in cancerassociated fibroblasts. Mol Cancer. 2017;16:148. https://doi.org/10.1186/ s12943-017-0718-4.

13. $\mathrm{Ma} \mathrm{P}$, et al. Extracellular vesicles-mediated noncoding RNAs transfer in cancer. J Hematol Oncol. 2017;10:57. https://doi.org/10.1186/s13045-017-0426-y.

14. Kim SH, Bianco NR, Shufesky WJ, Morelli AE, Robbins PD. MHC class II+ exosomes in plasma suppress inflammation in an antigen-specific and Fas ligand/Fas-dependent manner. J Immunol. 2007;179:2235-41. https://doi. org/10.4049/jimmunol.179.4.2235.

15. Seo N, Akiyoshi K, Shiku H. Exosome-mediated regulation of tumor immunology. Cancer Sci. 2018;109:2998-3004. https://doi.org/10.1111/cas.13735.

16. Chen G, et al. Exosomal PD-L1 contributes to immunosuppression and is associated with anti-PD-1 response. Nature. 2018;560:382-6. https://doi.org/ 10.1038/s41586-018-0392-8.

17. Greening DW, Gopal SK, Xu R, Simpson RJ, Chen W. Exosomes and their roles in immune regulation and cancer. Semin Cell Dev Biol. 2015;40:72-81. https://doi.org/10.1016/j.semcdb.2015.02.009.

18. Ristorcelli E, et al. Human tumor nanoparticles induce apoptosis of pancreatic cancer cells. FASEB J. 2008;22:3358-69. https://doi.org/10.1096/fj. 07-102855.

19. Theodoraki MN, et al. Circulating exosomes measure responses to therapy in head and neck cancer patients treated with cetuximab, ipilimumab, and IMRT. Oncoimmunology. 2019;8:1593805. https://doi.org/10.1080/2162402x. 2019.1593805.

20. Guo D, et al. Exosomes from heat-stressed tumour cells inhibit tumour growth by converting regulatory T cells to Th17 cells via IL-6. Immunology. 2018;154:132-43. https://doi.org/10.1111/imm.12874.

21. Yao Y, et al. Tumor cell-derived exosome-targeted dendritic cells stimulate stronger CD8+ CTL responses and antitumor immunities. Biochem Biophys Res Commun. 2013;436:60-5. https://doi.org/10.1016/j.bbrc.2013.05.058.

22. Rao Q, et al. Tumor-derived exosomes elicit tumor suppression in murine hepatocellular carcinoma models and humans in vitro. Hepatology. 2016;64: 456-72. https://doi.org/10.1002/hep.28549.

23. Zeelenberg IS, et al. Targeting tumor antigens to secreted membrane vesicles in vivo induces efficient antitumor immune responses. Cancer Res. 2008;68:1228-35. https://doi.org/10.1158/0008-5472.Can-07-3163.

24. Han Q, Zhao H, Jiang Y, Yin C, Zhang J. HCC-derived exosomes: critical player and target for Cancer immune escape. Cells. 2019;8. https://doi.org/ 10.3390/cells8060558.

25. Bu N, et al. Exosome-loaded dendritic cells elicit tumor-specific CD8+ cytotoxic T cells in patients with glioma. J Neuro-Oncol. 2011;104:659-67. https://doi.org/10.1007/s11060-011-0537-1.

26. Huang F, et al. TGF-beta1-silenced leukemia cell-derived exosomes target dendritic cells to induce potent anti-leukemic immunity in a mouse model. Cancer Immunol Immunother. 2017;66:1321-31. https://doi.org/10.1007/ s00262-017-2028-5.

27. Huang F, Wan J, Hu W, Hao S. Enhancement of anti-leukemia immunity by leukemia-derived exosomes via downregulation of TGF-beta1 expression. Cell Physiol Biochem. 2017:44:240-54. https://doi.org/10.1159/000484677.

28. $\mathrm{Xu} \mathrm{HY}$, et al. CD8+ T cells stimulated by exosomes derived from RenCa cells mediate specific immune responses through the FasL/Fas signaling pathway and, combined with GMCSF and IL12, enhance the antirenal cortical adenocarcinoma effect. Oncol Rep. 2019. https://doi.org/10.3892/or. 2019.7208.

29. Wang F, et al. Tumor-derived exosomes induce PD1(+) macrophage population in human gastric cancer that promotes disease progression. Oncogenesis. 2018;7:41. https://doi.org/10.1038/s41389-018-0049-3.

30. Boussadia Z, et al. Acidic microenvironment plays a key role in human melanoma progression through a sustained exosome mediated transfer of clinically relevant metastatic molecules. J Exp Clin Cancer Res. 2018;37:245. https://doi.org/10.1186/s13046-018-0915-z.

31. Shu S, et al. Publisher correction: metabolic reprogramming of stromal fibroblasts by melanoma exosome microRNA favours a pre-metastatic microenvironment. Sci Rep. 2019;9:4959. https://doi.org/10.1038/s41598018-37179-1.

32. Cianciaruso $\mathrm{C}$, et al. Molecular profiling and functional analysis of macrophage-derived tumor extracellular vesicles. Cell Rep. 2019;27:30623080.e3011. https://doi.org/10.1016/j.celrep.2019.05.008.

33. Zhang H, et al. CD4(+) T cell-released exosomes inhibit CD8(+) cytotoxic Tlymphocyte responses and antitumor immunity. Cell Mol Immunol. 2011;8: 23-30. https://doi.org/10.1038/cmi.2010.59. 
34. Seo N, et al. Activated CD8(+) T cell extracellular vesicles prevent tumour progression by targeting of lesional mesenchymal cells. Nat Commun. 2018; 9:435. https://doi.org/10.1038/s41467-018-02865-1.

35. Shen $M$, Ren X. New insights into the biological impacts of immune cellderived exosomes within the tumor environment. Cancer Lett. 2018;431: 115-22. https://doi.org/10.1016/j.canlet.2018.05.040

36. Zhang $F$, et al. Specific decrease in B-cell-derived extracellular vesicles enhances post-chemotherapeutic CD8+ T cell responses. Immunity. 2019. https://doi.org/10.1016/j.immuni.2019.01.010.

37. Zoller M. Janus-faced myeloid-derived suppressor cell exosomes for the good and the bad in Cancer and autoimmune disease. Front Immunol. 2018:9:137. https://doi.org/10.3389/fimmu.2018.00137.

38. Xie Y, Zhang X, Zhao T, Li W, Xiang J. Natural CD8(+)25(+) regulatory T cellsecreted exosomes capable of suppressing cytotoxic T lymphocytemediated immunity against B16 melanoma. Biochem Biophys Res Commun. 2013;438:152-5. https://doi.org/10.1016/j.bbrc.2013.07.044.

39. Andre $\mathrm{F}$, et al. Malignant effusions and immunogenic tumour-derived exosomes. Lancet. 2002;360:295-305. https://doi.org/10.1016/S01406736(02)09552-1

40. Wolfers J, et al. Tumor-derived exosomes are a source of shared tumor rejection antigens for CTL cross-priming. Nat Med. 2001;7:297. https://doi. org/10.1038/85438.

41. Lu Z, et al. Dendritic cell-derived exosomes elicit tumor regression in autochthonous hepatocellular carcinoma mouse models. J Hepatol. 2017;67: 739-48. https://doi.org/10.1016/j.jhep.2017.05.019.

42. Zhu $L$, et al. Exosomes derived from natural killer cells exert therapeutic effect in melanoma. Theranostics. 2017;7:2732-45. https://doi.org/10. 7150/thno.18752.

43. Shoae-Hassani A, et al. NK Cell-derived Exosomes From NK Cells Previously Exposed to Neuroblastoma Cells Augment the Antitumor Activity of Cytokine-activated NK Cells. J Immunother. 2017;40:265-76. https://doi.org/ 10.1097/cji.0000000000000179.

44. Sharma A. Role of stem cell derived exosomes in tumor biology. Int J Cancer. 2018;142:1086-92. https://doi.org/10.1002/ijc.31089.

45. Seo Y, Kim HS, Hong IS. Stem cell-derived extracellular vesicles as immunomodulatory therapeutics. Stem Cells Int. 2019;2019:5126156. https:// doi.org/10.1155/2019/5126156.

46. Reza A, Choi YJ, Yasuda H, Kim JH. Human adipose mesenchymal stem cellderived exosomal-miRNAs are critical factors for inducing anti-proliferation signalling to A2780 and SKOV-3 ovarian cancer cells. Sci Rep. 2016;6:38498. https://doi.org/10.1038/srep38498.

47. Li X, Liu LL, Yao JL, Wang K, Ai H. Human umbilical cord mesenchymal stem cell-derived extracellular vesicles inhibit endometrial Cancer cell proliferation and migration through delivery of exogenous miR-302a. Stem Cells Int. 2019;2019:8108576. https://doi.org/10.1155/2019/8108576.

48. Tung KH, Ernstoff MS, Allen C, Shu S. A review of exosomes and their role in the tumor microenvironment and host-tumor "macroenvironment". J Immunol Sci. 2019;3:4-8. https://doi.org/10.29245/2578-3009/2019/1.1165.

49. Ma J, et al. Exosomal hsa-miR199a-3p promotes proliferation and migration in neuroblastoma. Front Oncol. 2019;9:459. https://doi.org/10.3389/fonc. 2019.00459.

50. Ning $Y$, et al. Tumor exosomes block dendritic cells maturation to decrease the T cell immune response. Immunol Lett. 2018;199:36-43. https://doi.org/ 10.1016/j.imlet.2018.05.002.

51. Harada $\mathrm{K}$, et al. Tumor-associated macrophage infiltration is highly associated with PD-L1 expression in gastric adenocarcinoma. Gastric Cancer. 2018;21:31-40. https://doi.org/10.1007/s10120-017-0760-3.

52. Li $\mathrm{L}$, et al. Microenvironmental oxygen pressure orchestrates an anti- and pro-tumoral gammadelta T cell equilibrium via tumor-derived exosomes. Oncogene. 2019;38:2830-43. https://doi.org/10.1038/s41388-018-0627-z.

53. Liu J, et al. Endoplasmic reticulum stress causes liver Cancer cells to release Exosomal miR-23a-3p and up-regulate programmed death ligand 1 expression in macrophages. Hepatology. 2019. https://doi.org/10.1002/hep.30607.

54. Cheng $\mathrm{L}$, et al. Exosomes from melatonin treated Hepatocellularcarcinoma cells Alter the Immunosupression status through STAT3 pathway in macrophages. Int J Biol Sci. 2017;13:723-34. https:/doi.org/10.7150/ijbs.19642.

55. Haderk F, et al. Tumor-derived exosomes modulate PD-L1 expression in monocytes. Sci Immunol. 2017;2. https://doi.org/10.1126/sciimmunol.aah5509.

56. Qi J, et al. Exosomes derived from human bone marrow mesenchymal stem cells promote tumor growth through hedgehog signaling pathway. Cell Physiol Biochem. 2017:42:2242-54. https://doi.org/10.1159/000479998.
57. Kim DH, et al. Exosomal PD-L1 promotes tumor growth through immune escape in non-small cell lung cancer. Exp Mol Med. 2019;51:94. https://doi. org/10.1038/s12276-019-0295-2.

58. Theodoraki MN, et al. Clinical significance of PD-L1(+) exosomes in plasma of head and neck Cancer patients. Clin Cancer Res. 2018;24:896-905. https:// doi.org/10.1158/1078-0432.Ccr-17-2664.

59. Ludwig S, et al. Suppression of lymphocyte functions by plasma exosomes correlates with disease activity in patients with head and neck Cancer. Clin Cancer Res. 2017;23:4843-54. https://doi.org/10.1158/1078-0432.Ccr-16-2819.

60. Gordon SR, et al. PD-1 expression by tumour-associated macrophages inhibits phagocytosis and tumour immunity. Nature. 2017;545:495-9. https://doi.org/10.1038/nature22396.

61. Wei $Y$, et al. The local immune landscape determines tumor PD-L1 heterogeneity and sensitivity to therapy. J Clin Invest. 2019;130. https://doi. org/10.1172/JCl127726.

62. Pan BJ, et al. Correlation analysis of PD-L1 expression and prognosis in triple-negative breast cancers. Zhonghua Bing Li Xue Za Zhi. 2017:46:822-6. https://doi.org/10.3760/cma.j.issn.0529-5807.2017.12.002.

63. Wang H, et al. HIP1R targets PD-L1 to lysosomal degradation to alter T cellmediated cytotoxicity. Nat Chem Biol. 2019;15:42-50. https://doi.org/10. 1038/s41589-018-0161-x.

64. Abiko K, et al. IFN-gamma from lymphocytes induces PD-L1 expression and promotes progression of ovarian cancer. Br J Cancer. 2015;112:1501-9. https://doi.org/10.1038/bjc.2015.101.

65. Garcia-Diaz A, et al. Interferon receptor signaling pathways regulating PD-L1 and PD-L2 expression. Cell Rep. 2017;19:1189-201. https://doi.org/10.1016/j. celrep.2017.04.031.

66. Ruffell B, Coussens LM. Macrophages and therapeutic resistance in cancer. Cancer Cell. 2015;27:462-72. https://doi.org/10.1016/j.ccell.2015.02.015.

67. Qian X, et al. Respiratory hyperoxia reverses immunosuppression by regulating myeloid-derived suppressor cells and PD-L1 expression in a triple-negative breast cancer mouse model. Am J Cancer Res. 2019;9:529-45

68. Kasikara $C$, et al. Phosphatidylserine sensing by TAM receptors regulates AKT-dependent Chemoresistance and PD-L1 expression. Mol Cancer Res. 2017;15:753-64. https://doi.org/10.1158/1541-7786.Mcr-16-0350.

69. Moon PG, et al. Proteomic analysis of urinary exosomes from patients of early IgA nephropathy and thin basement membrane nephropathy. Proteomics. 2011;11:2459-75. https://doi.org/10.1002/pmic.201000443.

70. Poggio $M$, et al. Suppression of Exosomal PD-L1 induces systemic antitumor immunity and memory. Cell. 2019;177:414-427.e413. https://doi.org/ 10.1016/j.cell.2019.02.016

71. Yang $Y$, et al. Exosomal PD-L1 harbors active defense function to suppress T cell killing of breast cancer cells and promote tumor growth. Cell Res. 2018; 28:862-4. https://doi.org/10.1038/s41422-018-0060-4

72. Ricklefs FL, et al. Immune evasion mediated by PD-L1 on glioblastomaderived extracellular vesicles. Sci Adv. 2018;4:eaar2766. https://doi.org/10. 1126/sciadv.aar2766.

73. Razzo BM, et al. Tumor-derived exosomes promote carcinogenesis of murine oral squamous cell carcinoma. Carcinogenesis. 2019. https://doi.org/ 10.1093/carcin/bgz124.

74. Deng $\mathrm{L}$, et al. Irradiation and anti-PD-L1 treatment synergistically promote antitumor immunity in mice. J Clin Invest. 2014;124:687-95. https://doi.org/ 10.1172/jci67313.

75. van der Woude LL, Gorris MAJ, Halilovic A, Figdor CG, de Vries IJM. Migrating into the tumor: a roadmap for T cells. Trends Cancer. 2017;3:797808. https://doi.org/10.1016/j.trecan.2017.09.006.

76. Spranger S. Mechanisms of tumor escape in the context of the T-cellinflamed and the non-T-cell-inflamed tumor microenvironment. Int Immunol. 2016;28:383-91. https://doi.org/10.1093/intimm/dxw014

77. Gajewski TF. The next hurdle in Cancer immunotherapy: overcoming the non-T-cell-inflamed tumor microenvironment. Semin Oncol. 2015;42:663-71. https://doi.org/10.1053/j.seminoncol.2015.05.011.

78. Wei $Y$, et al. Efficacy and safety of combination immunotherapy for malignant solid tumors: a systematic review and meta-analysis. Crit Rev Oncol Hematol. 2019;138:178-89. https://doi.org/10.1016/j.critrevonc. 2019.04.008.

79. Yang Y, et al. Bispecific CD3-HAC carried by E1A-engineered mesenchymal stromal cells against metastatic breast cancer by blocking PD-L1 and activating T cells. J Hematol Oncol. 2019;12:46. https://doi.org/10.1186/ s13045-019-0723-8. 
80. Menzies AM, et al. Anti-PD-1 therapy in patients with advanced melanoma and preexisting autoimmune disorders or major toxicity with ipilimumab. Ann Oncol. 2017;28:368-76. https://doi.org/10.1093/annonc/mdw443.

81. Hartkopf AD, et al. PD-1 and PD-L1 immune checkpoint blockade to treat breast Cancer. Breast care (Basel). 2016;11:385-90. https://doi.org/10.1159/ 000453569.

82. Yamazaki N, et al. Phase 1b study of pembrolizumab (MK-3475; anti-PD-1 monoclonal antibody) in Japanese patients with advanced melanoma (KEYNOTE-041). Cancer Chemother Pharmacol. 2017;79:651-60. https://doi. org/10.1007/s00280-016-3237-x.

83. Kanz BA, et al. Safety and efficacy of anti-PD-1 in patients with baseline cardiac, renal, or hepatic dysfunction. J Immunother Cancer. 2016;4:60. https://doi.org/10.1186/s40425-016-0166-5.

84. Hamid $\mathrm{O}$, et al. Safety and tumor responses with lambrolizumab (anti-PD-1) in melanoma. N Engl J Med. 2013;369:134-44. https://doi.org/10.1056/ NEJMoa1305133.

85. Yu P, et al. Simultaneous inhibition of two regulatory T-cell subsets enhanced Interleukin-15 efficacy in a prostate tumor model. Proc Natl Acad Sci U S A. 2012;109:6187-92. https://doi.org/10.1073/pnas.1203479109.

86. Zhang Y, Zhou H, Zhang L. Which is the optimal immunotherapy for advanced squamous non-small-cell lung cancer in combination with chemotherapy: anti-PD-1 or anti-PD-L1? J Immunother Cancer. 2018;6:135. https://doi.org/10.1186/s40425-018-0427-6.

87. Gong B, et al. Secreted PD-L1 variants mediate resistance to PD-L1 blockade therapy in non-small cell lung cancer. J Exp Med. 2019;216:982-1000. https://doi.org/10.1084/jem.20180870.

88. Liu SY, et al. Sequential blockade of PD-1 and PD-L1 causes fulminant cardiotoxicity-from case report to mouse model validation. Cancers (Basel). 2019;11. https://doi.org/10.3390/cancers11040580.

89. Rosenzweig N, et al. PD-1/PD-L1 checkpoint blockade harnesses monocytederived macrophages to combat cognitive impairment in a tauopathy mouse model. Nat Commun. 2019;10:465. https://doi.org/10.1038/s41467019-08352-5.

90. Guo Y, et al. Effects of exosomes on pre-metastatic niche formation in tumors. Mol Cancer. 2019;18:39. https://doi.org/10.1186/s12943-019-0995-1.

91. Raeven P, Zipperle J, Drechsler S. Extracellular vesicles as markers and mediators in sepsis. Theranostics. 2018;8:3348-65. https://doi.org/10.7150/ thno.23453.

92. Lux A, Kahlert C, Grutzmann R, Pilarsky C. C-met and PD-L1 on circulating exosomes as diagnostic and prognostic markers for pancreatic Cancer. Int J Mol Sci. 2019;20. https://doi.org/10.3390/ijms20133305

93. Yang $Y$, et al. An Immuno-biochip selectively captures tumor-derived exosomes and detects Exosomal RNAs for Cancer diagnosis. ACS Appl Mater Interfaces. 2018;10:43375-86. https://doi.org/10.1021/acsami.8b13971.

\section{Publisher's Note}

Springer Nature remains neutral with regard to jurisdictional claims in published maps and institutional affiliations.

Ready to submit your research? Choose BMC and benefit from:

- fast, convenient online submission

- thorough peer review by experienced researchers in your field

- rapid publication on acceptance

- support for research data, including large and complex data types

- gold Open Access which fosters wider collaboration and increased citations

- maximum visibility for your research: over $100 \mathrm{M}$ website views per year

At BMC, research is always in progress.

Learn more biomedcentral.com/submissions 